\title{
Comment on bulk excitons in solid neon: Experiment
}

\author{
V. Saile and E. E. Koch \\ Hamburger Synchrotronstrahlungslabor, HASYLAB, Deutsches Elektronen-Synchrotron DESY, \\ 2000 Hamburg 52, Germany \\ (Received 27 August 1979)
}

\begin{abstract}
We compare experimental results from absorption, transmission, and reflection experiments for solid neon in order to facilitate comparison with theory. Further we point out that the relative intensities of the spin-orbit split $n=1$ excitons in solid rare gases can differ significantly from those of the corresponding atomic transitions.
\end{abstract}

In the preceding paper, Resca and Resta comment on our experimental results for solid neon. ${ }^{1}$ They point out that the $n=1$ excitons are found at lower energies than in our previously reported reflection spectra. ${ }^{2}$ While the theoretical results ${ }^{3-5}$ are in perfect agreement with the experimental exciton energies observed in reflectivity, ${ }^{2}$ the $n=1$ excitons occur at lower energies in transmission ${ }^{1}$ and additional assumptions on the effective-mass parameter have been suggested by Resca and Resta in order to obtain a better agreement with the transmission data.

To elucidate the situation, we compare in Table I the $n=1 \quad\left(j=\frac{1}{2}\right)$ energies published in the past few years ${ }^{6}$ and compare these with our results. ${ }^{1,2}$ Table I clearly demonstrates that our results are within the

TABLE I. Experimental $n=1 \quad\left(j=\frac{1}{2}\right)$ energies and derived values for the imaginary part $\epsilon_{2}$ of the dielectric function compared to the reflection and transmission results in Refs. 2 and 1.

\begin{tabular}{ccc}
\hline \hline Reflection & $\begin{array}{c}\epsilon_{2} \text { from reflection } \\
\text { with Kramers-Kronig } \\
\text { analysis }\end{array}$ & Absorption, transmission \\
\hline $17.80^{\mathrm{a}}$ & $17.49^{\mathrm{b}}$ & $17.4^{\mathrm{a}}$ \\
$17.83^{\mathrm{c}}$ & $17.59^{\mathrm{c}}$ & $17.48^{\mathrm{d}}$ \\
$17.79^{\mathrm{e}}$ & & $17.50^{\mathrm{f}}$ \\
\hline \hline
\end{tabular}

${ }^{a}$ R. Haensel, G. Keitel, E. E. Koch, N. Kosuch, and M. Skibowski, Phys. Rev. Lett. 25, 1281 (1970).

${ }^{b} \mathrm{M}$. Skibowski et al., Third International Conference on

VUV Radiation Physics, Tokyo, 1971 (unpublished), as cited in Ref. 6.

${ }^{\text {cReference } 8 .}$.

${ }^{d}$ E. Boursey, J. -Y. Roncin, and H. Damany, Phys. Rev. Lett. 25, 1279 (1970)

${ }^{\text {e}}$ Reference 2.

fReference 1. accuracy of the different experiments in full agreement with the results previously reported. We note that the difference between the peak positions derived from absorption and reflection measurements reflect the fact, that in an absorption experiment maxima in $\epsilon_{2}$ are monitored whereas a reflection spectrum has its maximum between the transverse and longitudinal frequency of the exciton band. The connection between both optical constants can be established via a Kramers-Kronig analysis (see Table I).

The assignment of the strongest peak to $n=1$ $\left(j=\frac{1}{2}\right)$ is based on the analogy with the free atom, where $2 p^{6} \rightarrow 2 p^{5} 3 s^{\prime}\left(j=\frac{1}{2}\right)$ is very strong while $2 p^{6} \rightarrow 2 p^{5} 3 s\left(j=\frac{3}{2}\right)$ is suppressed due to selection rules. If we take the atomic $2 p^{6} \rightarrow 3 s, 3 s^{\prime}$ separation which includes spin-orbit interaction and exchange effects, we expect the $n=1\left(j=\frac{3}{2}\right)$ transition at $17.32 \mathrm{eV}$ which is rather close to the observed value of $17.36 \mathrm{eV} .^{1}$ Admittedly the analysis of the wellresolved transmission spectrum between 17 and 18 $\mathrm{eV}$ for $\mathrm{Ne}$ is quite complicated because obviously at least four transitions overlap strongly. The best solution to that experimental problem is, to our knowledge, a combination of transmission and reflection spectroscopy as applied in Ref. 1 .

We further comment on the intensities of the exciton bands. In a recent publication ${ }^{7}$ Resca and Resta modified within their quantum defect approach the Wannier formula for intensity ratios of the $n$th member of the series to the first one from

$$
\frac{I_{n}}{I_{1}}=n^{-3}
$$

to

$$
\frac{I_{n}}{I_{1}}=(n+\delta)^{-3}
$$

with a quantum defect $\delta$. As demonstrated in their work, Eq. (2) yields much better results than Eq. (1) when compared with experiment. ${ }^{8}$ We want to direct 
TABLE II. Comparison between intensity ratios for the $n=1$ excitons in rare-gas solids and the corresponding atomic transitions.

\begin{tabular}{|c|c|c|c|c|c|}
\hline & & $\mathrm{Ne}$ & $\mathrm{Ar}$ & $\mathrm{Kr}$ & $\mathrm{Xe}$ \\
\hline \multirow{2}{*}{ Solid } & $I\left(n=1, j=\frac{3}{2}\right)$ & \multirow{2}{*}{$1: 5-1: 10$} & \multirow{2}{*}{$1: 1$} & \multirow{2}{*}{$1: 1$} & \multirow{2}{*}{-} \\
\hline & $I\left(n=1, j=\frac{1}{2}\right)$ & & & & \\
\hline \multirow{3}{*}{ Atom $(e)$} & & a & b & c & d \\
\hline & $n p^{6} \rightarrow n p^{5}(n+1) s$ & \multirow[t]{2}{*}{$\leqslant 1: 10$} & \multirow{2}{*}{$1: 3-1: 4.4$} & \multirow[t]{2}{*}{$1: 1$} & \multirow[t]{2}{*}{$1: 1-1: 0.8$} \\
\hline & $\overline{n p^{6} \rightarrow n p^{5}(n+1) s^{\prime}}$ & & & & \\
\hline
\end{tabular}

\footnotetext{
${ }^{\mathrm{a}}$ Reference 1.

${ }^{b}$ A. Harmsen, E. E. Koch, V. Saile, N. Schwentner, and M. Skibowski, in Vacuum Ultraviolet Radiation Physics, edited by E. E. Koch, R. Haensel, and C. Kunz (Pergamon/Vieweg, Braunschweig, 1974), p. 339; V. Saile, thesis (Universität Müchen, 1976) (unpublished).

'S. S. Hasnain, T. D. S. Hamilton, and I. H. Munro, J. Phys. C $\underline{11}$, L261 (1978); see also Ref. 6. ${ }^{\mathrm{d}}$ The $n=1 \quad\left(j=\frac{1}{2}\right)$ exciton is observed above the band edge. This degeneracy with the continuum of $j=\frac{3}{2}$ states prevents a simple estimate of the strength of this transition.

${ }^{\mathrm{e}} \mathrm{A}$ collection of experimental and theoretical values can be found in Ref. 9.
}

attention to a limitation of this approach: Equation (2) is generally only able to predict the progression of oscillator strengths of the whole multiplet with quantum number $n .^{9}$ This means, that the strengths of corresponding members with the same $n$ of the $j=\frac{1}{2}$ and $\frac{3}{2}$ series have to be added to obtain $I_{n}$. In general, the internal distribution of oscillator strengths within the multiplet turns out to be different in the atom and solid rare gas. This is illustrated in Table II for the $n=1$ excitons. At least for Ar and probably for Ne solid-state effects beyond dielectric screening modify considerably the atomic wave functions.

Finally, we compare in Table III our experimental results with the theoretical data published by Martinelli et al. ${ }^{10}$ and Grosso et al. ${ }^{11}$ In both papers the integral equation approach for excitons ${ }^{12}$ is applied to an isolated $\mathrm{Ne}$ atom. This approach leads to a Schrödinger equation for the envelope functions with an appropriate Coulomb potential and a semiempirical repulsive potential due to orthogonalization effects. The parameter for the repulsive potential is adjusted using known atomic excitations in $\mathrm{Ne}$. The effective mass and an electron-hole polarization potential have to be included in the Hamiltonian for the calculation of exciton energies. For this purpose a model potential similar to the one used in the $F$ center problem is used in Refs. 10 and 11. The final numerical results obtained in Refs. 10 and 11 differ slightly and both are not fully in accord with experiment. The discrepancy between the theoretical results seems to be due to different values used for the short-range part of the electron-hole polarization potential which differs by a factor of 0.5 . We note at this point, however, that the importance and influence of dielectric screening in the solid is demonstrated in a convincing way in Refs. 10 and 11.

TABLE III. Comparison between experiment and theoretical results by Martinelli et al. (Ref. 10) and Grosso et al. (Ref. 11) for the $j=\frac{1}{2}$ series in solid Ne.

\begin{tabular}{cccccc}
\hline \hline$n$ & 1 & 2 & 3 & 4 & 5 \\
\hline Experiment $^{\mathrm{a}}$ & 17.36 & 20.25 & 20.94 & 21.19 & 21.32 \\
Theory $^{\mathrm{b}}$ & 17.37 & 20.64 & 21.19 & 21.40 & 21.50 \\
Theory $^{\mathrm{c}}$ & 17.93 & 20.62 & 21.17 & 21.38 & 21.49 \\
\hline \hline
\end{tabular}

\begin{tabular}{llc}
\hline a Reference 1. & ${ }^{\mathrm{b}}$ Reference 10. & 'Reference 11.
\end{tabular}




\section{ACKNOWLEDGMENTS}

We want to thank L. Resca and R. Resta for providing their comment prior to publication and for bringing to our attention Refs. 10 and 11 which were not included in our previous paper (Ref. 1).

'V. Saile and E. E. Koch, Phys. Rev. B 20, 784 (1979).

${ }^{2}$ V. Saile, W. Steinmann, and E. E. Koch, in Extended Abstracts of the 5th International Conference on Vacuum Ultraviolet Radiation Physics, Montpellier, 1977, edited by M. C. Castex, M. Pouey, and N. Pouey (CNRS, Meudon, 1977), p. 199.

${ }^{3}$ R. Resta, Phys. Status Solidi B 86, 627 (1978).

${ }^{4}$ L. Resca, R. Resta, and S. Rodriguez, Solid State Commun. 26, 849 (1978).

${ }^{5}$ L. Resca, R. Resta, and S. Rodriguez, Phys. Rev. B $\underline{18}, 696$ (1978).

${ }^{6}$ B. Sonntag, in Rare Gas Solids, edited by M. L. Klein and J. A. Venables (Academic, New York, 1977), Vol. II, p. 1021
${ }^{7}$ L. Resca and R. Resta, Phys. Rev. B $\underline{19}, 1683$ (1979).

${ }^{8}$ D. Pudewill, F. -J. Himpsel, V. Saile, N. Schwentner, M. Skibowski, and E. E. Koch, Phys. Status Solidi B 4ㅡ, 485 (1976).

${ }^{9}$ P. S. Ganas and A. E. S. Green, Phys Rev. A $\underline{4}, 182$ (1971).

${ }^{10}$ L. Martinelli and G. Pastori Parravicini, J. Phys. C $\underline{10}$, L687 (1977).

${ }^{11}$ G. Grosso, L. Martinelli, and G. Pastori Parravicini, Solid State Commun. 25, 435 (1978).

${ }^{12} \mathrm{~W}$. Andreoni, M. Altarelli, and F. Bassani, Phys. Rev. B 11, 2352 (1975). 